\title{
La Confederación Nacional de Estudiantes Católicos: orígenes, primeros pasos y consolidación (1920-1923)
}

\author{
Donato Barba Prieto
}

\section{RESUMEN}

Dentro de la Asociación Católica Nacional de Propagandistas, fundada por el padre Ayala en los años finales de la primera década del siglo $X X$, se promueven diversas fómulas de participación de los católicos en la vida pública española, una de las cuales va a ser la de los Estudiantes Católicos, cuyo ámbito será el mundo universitario, si bien intentará más adelante desarrollar también su labor en los centros de Bachillerato. Aunque la vida de la Confederación comprende los años 1920 a 1939, se recogen en este trabajo algunos aspectos de sus primeros años (19201923), como su fundación en 1920, el crecimiento y desarrollo manifestado en

la Primera Asamblea Confederal de Zaragoza, el enfrentamiento con otras asociaciones de estudiantes o su presencia relevante en organizaciones internacionales como Pax Romana.

PALABRAS CLAVE Catolicismo, Universidad, Asociación Católica Nacional de Propagandistas,

\section{ABSTRACT}

Several formulae of participation of Roman Catholics in public life in Spain were originated within the Catholic National Association of Propagandistas, founded by father Ayala towards the end of the first decade of twentieth century; one of such was the National Confederation of Catholic Students.

The scope of this confederation was targeted primarily to the university world, but later on it was succesfully extended also to high schools.

Although the life span of the Confederation comprises the years 1920 until 1939, this paper covers some aspects of the early period only (1920-1923); namely its birth in 1920 , growth and development as being manifest during the First Confederal Assambly in Zaragoza; its confrontations with different students' associations, and its relevant role in international organizations, i. e. Pax Romana. 


\section{INTRODUCCIÓN}

Surgen los Estudiantes Católicos a comienzos de los años veinte con el deseo de afrontar la crisis de las instituciones universitarias y con el trasfondo de inyectar savia cristiana a la sociedad española de la época. Sus miembros ingresan en la Confederación en los años de universidad y muchos mantienen su vinculación con la institución después de acabados sus estudios.

Su actividad coincide con los últimos años del reinado constitucional de Alfonso XIII, la Dictadura de Primo de Rivera y la II República; la Guerra paralizó su actividad y un intento de resucitar la Confederación, apoyado por el cardenal Gomá, no tuvo eco en Franco, que abortó cualquier iniciativa que no pudiera controlar directamente, dejando como única posibilidad su integración - disolución - en el SEU.

Además los Estudiantes Católicos formaban parte de un proyecto más amplio de participación social y política, en consonancia con las directrices de la Santa Sede, consistente en animar a los católicos a intervenir en la vida pública; esto tendría su reflejo en el campo en la creación del Partido Social Popular, si bien el escaso tiempo del que dispuso para desarrollar su actividad, impidió consolidarse como el primer partido democristiano en España, que hubiera contado con el respaldo, entre otros, de los Estudiantes Católicos.

En este trabajo sólo vamos a estudiar la fase inicial de la historia de los Estudiantes Católicos, en la que se forjan las líneas básicas de actuación y de organización y en la que la Confederación experimenta ya un crecimiento notable. Es interesante subrayar el hecho de que este primer crecimiento y desarrollo fue anterior al advenimiento de la Dictadura y no fue, por tanto, consecuencia de la llegada de ésta.

\section{ALGUNOS PROBLEMAS DE LA UNIVERSIDAD ESPAÑOLA EN LA RESTAURACIÓN}

En torno a la Universidad española durante la Restauración, y tomando como referencia los aspectos ideológicos, podemos hablar de dos grandes grupos; uno en torno a la Institución Libre de Enseñanza, agrupando a esas tres generaciones de las que hablará Ortega: abuelos, contemporáneos de Francisco Giner de los Ríos, hijos y nietos. Estos últimos conectaron mucho mejor con el "abuelo" y tuvieron además una presencia pública muy notable: Azaña, Pijoán, Ortega, Luzu- 
riaga, Marañón, Onís, Madariaga, García Morente, Jiménez Fraud, Julio Camba, Juan Ramón Jiménez, Pérez de Ayala, Américo Castro, Jiménez de Asúa, Augusto Barcia... '.

El otro sector tomaba como punto de referencia a Menéndez Pelayo, si bien sus discípulos eran menos numerosos que los de Giner y sus obras también lo eran. Quizás sirvió este grupo como aglutinante del pensamiento católico en dos ideas: cristianismo y tradición.

Ambos grupos, que pretendian la mejora del sistema educativo poniendo coto al analfabetismo, propugnando un aumento del número de escuelas y la formación de buenos maestros e investigadores, tenian, no obsiante, opiniones contrapuestas sobre materias tales como «libertad de cátedra" o "autonomía universitaria". Desde luego las posiciones de "reformadores" y "tradicionales" sobre estos temas estaban claras. Para los primeros la libertad de cátedra era algo irrenunciable, pieza clave de la reforma de la enseñanza y única manera de luchar contra el inmovilismo social y cultural. En primer plano se colocan los ideales de progreso y libertad, y, como consecuencia, cualquier política educativa debería estar apoyada sobre la libertad científica y la secularización del saber. No se le podia exigir a ningún catedrático que enseñase ninguna doctrina que estuviese en contra de su conciencia, aunque fuese el dogma de la religión del Estado; desde este punto de vista la secularización se interpretaba en el sentido de apartar a la Iglesia de cualquier labor docente.

En el otro campo, el de los "tradicionales", la libertad de cátedra debía tener como guía el conocimiento de la verdad, la adhesión a una serie de principios incuestionables a los que habían de someterse las inteligencias, y, por eso, la defensa de la verdad pasaba por la supervisión de la Iglesia sobre la enseñanza.

Algo parecido ocurría con la autonomía universitaria; mientras unos eran partidarios del mantenimiento del centralismo universitario, otros se decantaban por la puesta en marcha de una verdadera autonomía universitaria como medio para conseguir la revitalización de la Universidad.

$Y$ no cabe duda que los aspectos políticos no estaban exentos de estos debates, ya que tras la crisis del gobierno Romanones, subió al poder Maura, el 15 de abril de 1919, y con él, como ministro de Instrucción Pública llegó César Silió, que se puso como uno de los objetivos de su ministerio el sacar adelante el proyecto de autonomía para las universidades, 
que efectivamente vio la luz con el Real Decreto de 21 de mayo de ese mismo año, es decir, poco más de un mes después de llegar al gobierno.

Por otra parte a partir de 1917 hay una efervescencia universitaria que corre en paralelo con la crisis política, militar, regionalista y social que se produce en toda España. En algunos lugares los estudiantes se unen a las masas obreras, como en el caso de Granada, en los incidentes del 11 de febrero de 1919, en los que murieron tres personas, entre ellas un estudiante ${ }^{2}$.

En este contexto no hace falta resaltar, por obvio, la importancia del Real Decreto del 21 de mayo de 1919, que dio luz verde a la autonomía universitaria, que impulsaba la participación de los estudiantes en la vida universitaria, incluyendo la elección de cargos docentes. Marcaba también las líneas generales que las asociaciones deberian seguir, sobre todo en lo referente a la participación en los claustros extraordinarios, condiciones de aprobación, número mínimo de afiliados... No cabe duda que la relevancia que se le daba a las asociaciones estudiantiles iba a repercutir en su proliferación.

Así surgirán la Confederación Nacional de Estudiantes Católicos y la Unión Nacional de Estudiantes, próxima a la Institución Libre de Enseñanza. También aparecerá por esta época una agrupación de estudiantes socialistas, cuya importancia fue pequeña.

\section{LOS ORIGENES DE LA CONFEDERACIÓN NACIONAL DE ESTUDIANTES CATÓLICOS}

El padre Ayala, fundador de la Asociación Nacional de Propagandistas, comentaba en el verano de 1930:

«No sé cuando ideé la ACN. de P. Mi propósito era reunir jóvenes para hacer algo..., para dar mítines. En cuanto a los estudiantes, la parte que tomé fuera de la obsesión de que no se adelantasen en la organización los neutros, consistió en que un dia, leyendo en El Debate la reseña de un discurso de Fernando Martín-Sánchez, pensé que era el indicado para hacer la Confederación. No me equivoqué. Fernando es realmente el padre de la Obra de los Estudiantes Católicos" " 3 .

Y, efectivamente, Fernando Martín Sánchez fue el que concretó la intuición del padre Ayala. Él mismo, en un acto de homenaje al padre Ayala

Cfr. Calero AMOR, Antonio: Historia del movimiento obrero en Granada (1909-1923), p. 106 Boletín de la ACN. de P., 5 de julio de 1930. 
celebrado el 22 de junio de 1930, contó que en 1920 se recibió una carta de Friburgo invitando a España a sumarse a una Internacional de Estudiantes Católicos que se proyectaba fundar. Esto hizo nacer la idea que hacia tiempo habia germinado en la mente del padre Ayala ${ }^{4}$.

Un poco antes, en mayo de 1919, había publicado El Debate un comentario sobre la aparición de una organización escolar socialista en Valladolid, lo que aprovechaba el redactor para proclamar la necesidad de crear asociaciones estudiantiles católicas ${ }^{5}$. Quizá esté aqui el origen próximo de la fundación de los Estudiantes Católicos.

Como el 21 de mayo de ese mismo año se publica el Decreto Silió que posibilita la creación de organizaciones de estudiantes, los propagandistas se pusieron a organizarse y en la Asamblea General de la Asociación que se celebró en Loyola, en septiembre de 1919, se urgió a concretar todo esto. Y así se llega al 7 de marzo de 1920, fiesta entonces de Santo Tomás de Aquino, día en que se reune una Junta Nacional organizadora que decide constituir la Confederación Nacional de Estudiantes Católicos de España ${ }^{6}$. No obstante, no se puede hablar todavia de una realidad, sino más bien de una declaración de intenciones, ya que El Debate del día 9 de marzo, es decir, dos días después de celebrada la reunión de la Junta Nacional, expresa la necesidad de constituir la Asociación Nacional de Estudiantes Católicos de España. Así, de una manera informal y sencilla - como habia ocurrido con la misma ACN. de $P$. allá por 1908- se irán dando mítines y surgirán asociaciones de estudiantes católicos, primero en los núcleos de población más próximos a Madrid: Toledo, Ávila, Segovia, Guadalajara y luego en otras ciudades universitarias como Valencia, Zaragoza, Valladolid, Granada...

El Debate recoge ya el 14 de marzo la celebración de un mitin de estudiantes católicos celebrado en Ávila y en el que intervinieron los estudiantes Luis Sierrra, José María Pérez Laborda — futuro presidente de la Juventud de Acción Popular-y Fernando Martín-Sánchez. Además de la reseña del acto, el periódico le dedica un artículo de fondo con el título de Una empresa transcendental, que termina con estas palabras:

"Por eso nosotros saludamos a la nueva Asociación como un acontecimiento de verdadera transcendencia en la vida nacional» ?

\footnotetext{
4 Cfr. Martin Martinez, Isidoro: Ángel Herrera y la enseñanza, en memoria del centenario del nacimiento de Ángel Herrera, 30 de octubre de 1987.

5 Cfr. El Debate, 15 de mayo de 1919.

6 Cfr. Memoria sobre la Confederación Nacional de Estudiantes Católicos, elaborada por Martín Artajo, recogida en Juventud católica española, vol. III, 1927.

martin Martinez, Isidoro, op. cit.
} 


\section{EL MITIN DE LA ZARZUELA, "EPIFANIA "DE LA CONFEDERACIÓN}

El 13 de mayo de 1920 tuvo lugar en el teatro de la Zarzuela de Madrid un gran mitin que será muy importante para el futuro de la Confederación y que algunos de los dirigentes de los propagandistas, siguiendo al redactor de El Debate, gustarán llamar "epifania», es decir, la manifestación pública de esos primeros pasos dados dos meses antes. El Debate recogió la noticia al día siguiente. Presidió el acto el señor Artiñano, catedrático de la Escuela de Ingenieros Industriales. Había numerosos estudiantes, representantes de Valencia, Granada, León, Segovia, Ávila, Guadalajara, Badajoz y Madrid.

El primero en tomar la palabra fue Marcelino Oreja, de la Escuela de Ingenieros de Caminos, que fue el primer secretario de la Confederación. A continuación intervino Yanguas Messía, catedrático de la Facultad de Derecho, que sería más adelante presidente de la Asamblea Nacional y ministro de Estado con Primo de Rivera. Cerró el acto el señor Artiñano, leyéndose al final una carta de adhesión de Vázquez de Mella ${ }^{8}$.

Como conclusión del acto se elaboró un amplio programa que, en forma de manifiesto se elevó al Ministro de Instrucción Pública. Se componía de quince peticiones entre las que se encontraban como más destacadas la libertad de enseñanza, la necesidad de poner límites a la libertad de cátedra, la concesión de una amplia autonomía universitaria y el derecho de los estudiantes a participar en la vida universitaria ${ }^{9}$.

\section{ESTRUCTURA ORGANIZATIVA Y PRIMERAS ACTIVIDADES}

Poco a poco el movimiento estudiantil católico fue creciendo y se estructuró en una organización central, regional y local, que permitió aunar los esfuerzos y aumentar la eficacia.

La base de la obra de los estudiantes católicos fue la Asociación, que consistía en la agrupación de estudiantes de una misma carrera o especialidad en una escuela o facultad universitaria: Derecho, Medicina, Cien-

8 Cfr. El Debate, 14 de mayo de 1920.

Cfr. Hoja de imprenta para información y propaganda. Estas peticiones, aunque de manera más breve las recogen también GonzÁlez RuIz, Nicolás y MARTin MARTínez, Isidoro en su obra Seglares en la Historia del catolicismo español. 
cias, Magisterio, Bachillerato... Ellos mismos elegian la dirección que les gobernaría durante ese curso ${ }^{10}$.

Al finalizar el curso 1920-1921 el número de Asociaciones era de 32, con un número aproximado de 3.500 socios. Ya en el curso siguiente, 1921-1922, se produjo un notable incremento: 69 Asociaciones -más del doble - y unos 14000 asociados - cuatro veces más-. No cabe duda de que la implantación de las Asociaciones de Estudiantes católicos era un hecho incuestionable.

No obstante, en el curso 1922-23, último que consideramos en este trabajo, se ha producido un descenso: 61 Asociaciones y unos 13.200 miembros. Estos vaivenes pueden explicarse por la renovación de los cargos directivos o por el simple hecho de concluir sus estudios y darse de baja algunos estudiantes.

¿Cuál fue la labor que llevaron a cabo estas Asociaciones? En palabras de Martín Artajo hicieron mucho en favor de

"la formación religiosa y social de los escolares, de la elevación del nivel de cultura del estudiante, de la dignificación de la clase ${ }^{11}$, de su bienestar" ${ }^{12}$.

El conjunto de las diversas Asociaciones de cada localidad o región constituye la Federación. Su labor es variada y abarca campos como la formación religiosa (cursos de cultura apologética, por ejemplo), actividades literarias y científicas (Bibliotecas, Ateneos, certámenes, revistas, conferencias...), etc. El centro de estas actividades es la Casa del Estudiante, domicilio habitual de la Federación y de las Asociaciones, aunque éstas puedan tener otro en la propia escuela o facultad correspondiente.

La Federación tiene su Junta Federal y su Consejo (formado éste por profesores y personas relevantes) y un Consiliario con funciones que hoy diríamos de capellán. El Consejo Federal trata de las cuestiones más graves y tiene función asesora. El Consiliario cuida de que la actividad desarrollada por las Asociaciones, tanto en la orientación general como en la resolución de algún problema concreto, no exceda de los límites de la moral o de la doctrina cristiana.

10 Cfr. Memoria sobre la Confederación Nacional de Estudiantes Católicos, pp. 196 y ss., elaborada por Alberto MARTIN ARTAJo y recogida en Juventud católica española, vol. III, 1927; en general para todo lo referente a la organización seguiremos esta fuente.

1 Según el propio MARTín ARTAJO, los estudiantes constituyen una clase, son a modo de sindicatos, lo que es lo mismo que decir que tienen intereses comunes a todos ellos; resulta así lógica su asociación, para conseguir la fuerza que da la unión.

12 Ibidem, pp. 219-220. 
El número de Federaciones fue de 15 en el curso 1920-1921, 22 en el de 1921-1922 y 21 en el curso 1922-1923. Algunas Federaciones que tuvieron gran pujanza en los años 1920-1922 desaparecieron después, caso de las de Oviedo y Santiago. La oposición frontal del profesorado hizo quebrar a la primera y el carácter fuertemente individualista de los estudiantes de Santiago dio al traste con la segunda. Estas dos, junto a Murcia eran las únicas ciudades universitarias donde no existían en 1923 los Estudiantes Católicos.

Aportando datos del archivo de la secretaría confederal, señala Martín Artajo que durante el curso 1921-1922 organizaron cursos de conferencias, para todos los estudiantes en general, las Federaciones de Madrid, Santiago, Valencia y Zaragoza; y de las restantes Federaciones la que menos celebró durante ese curso fueron siete conferencias, todas ellas a cargo de catedráticos y otras personalidades. Además se inaugura el círculo de estudios de la Confederación y funcionan ya otros varios en las Federaciones ${ }^{13}$. Se celebraron diez o doce veladas literarias en distintas Asociaciones y se publicaron regularmente algunas revistas como Libertas en Valencia, Ilustración Española en Burgos, Santillana en Santander o Salmántica en Salamanca y se organiza una Exposición Escolar Hispanoamericana de pintura en Madrid.

Las Federaciones procuran también ayudar a sus miembros en materia económica, creando una "Comisión de ventajas materiales" que gestiona rebajas en algunos artículos propios del estudiante, especialmente espectáculos, deportes, exposiciones artísticas, viajes...; además, las Federaciones con una economía más saneada sostienen algunas becas, como es el caso de la de Valencia o la de Salamanca.

El órgano de gobierno central es la Confederación, que en palabras de Martín Artajo constituye la unidad espiritual de todos los Estudiantes Católicos Españoles ${ }^{14}$. Abarca a todas las Federaciones y da unidad a toda la estructura. Su actividad abarca tres facetas: dirección de la estrategia a seguir en las grandes campañas escolares nacionales, representación de los Estudiantes Católicos ante los poderes públicos y los organismos internacionales, y, por último, creación y organización de Asociaciones y Federaciones por toda España.

Se gobierna por una Junta Suprema elegida por un año por la Asamblea General, que se celebra anualmente. Como organismo asesor cuen-

13 Los círculos de estudios son reuniones, normalmente semanales, en los que se expone y asimila la doctrina social de la Iglesia. Comenzaron a darse en 1914, en el seno de lo que entonces se llamaba la Asociación Católica Nacional de Jóvenes Propagandistas.

14 lbidem, p. 198. 
ta con un Comité de antiguos estudiantes y con un Consejo Confederal formado por personalidades relevantes del mundo universitario, científico y de la Acción Católica.

Tras el ya citado mitin de la Zarzuela del día 13 de mayo de 1920 en Madrid, en el que se anuncian los principios fundamentales que se irán desarrollando con posterioridad, los actos promovidos por la Confederación van en esa línea; así el 30 de enero de 1921 se celebró en Madrid un acto escolar, presidido por el Rector de la Universidad Central, en el que quedó clara la postura de la Confederación favorable a la autonomía universitaria.

Un hecho que resultará muy significativo en la trayectoria de la Confederación, será la Fiesta Escolar que se celebró el 14 de marzo de 1921 en el Paraninfo de la Universidad de Valencia, presidida por el arzobispo Dr. Reig, para conmemorar a Santo Tomás de Aquino, antecedente inmediato de la Fiesta del Estudiante, que a partir del año siguiente se celebraría el 7 de marzo en toda España.

También en 1921 asistirá Fernando Martín-Sánchez a la fundación de Pax Romana, la Internacional de Estudiantes Católicos, siendo elegido vicepresidente e ingresando España en dicha organización.

Al año siguiente, en febrero de 1922, hay un debate público sobre la confesionalidad celebrado en la Universidad de Salamanca, siendo el ponente y ardoroso defensor de la misma José María Gil Robles ${ }^{15}$.

Por fin, del 20 al 23 de enero de 1923 tiene lugar en la Universidad de Zaragoza la I Asamblea Nacional de la Confederación, que estudiaremos a continuación.

\section{LA PRIMERA ASAMBLEA CONFEDERAL DE ZARAGOZA}

Al ser la primera reunión de estas características que celebraron los Estudiantes Católicos, los temas que se iban a tratar eran, si podemos hablar así, los "fundacionales": necesidad de la confesionalidad, qué debe ser una Asociación de Estudiantes Católicos, así como otros asuntos prácticos del estilo de las cuotas que se deben pagar para mantener la Confederación, o cómo intercambiar información y propaganda entre las Federaciones ${ }^{16}$.

\footnotetext{
Cfr. Memoria..., pp. 211-212, donde se recogen algunos de los actos más significativos

16 Cir. Obras Fecundas. Primera Asamblea de la Confederación Nacional de Estudiantes Católicos. Zaragoza, 1923.
} 
La Asamblea comenzó con un día de retiro y las sesiones tendrían lugar en los locales universitarios que a este fin facilitó el Rector de la Universidad de Zaragoza, Royo Villanova.

Asistieron delegados de 70 Asociaciones ${ }^{17}$. Se leyeron las adhesiones: saludo de Alfonso XIII, elogiosa carta del nuncio Tedeschini, apoyo de Maura... Tras esto tomó la palabra el presidente de la Confederación, Fernando Martín-Sánchez, quien después de los saludos y agradecimientos protocolarios, esbozó el programa de la Confederación:

"La Confederación Nacional de Estudiantes Católicos es una organización escolar profesional confesional. Profesional, es decir, que se ocupa de la profesión de sus miembros, de la enseñanza. No es nuestro fin organizar actos religiosos ni conferencias ni veladas; para cada una de estas cosas existen Congregaciones, Ateneos y Cuadros artísticos. Nuestro fin es ocuparnos de la enseñanza en todos sus aspectos, y del estudiante, como sujeto paciente de ella... y sólo con la enumeración de este nuestro fin profesional surge la necesidad de lo confesional. Para actuar en materia de enseñanza es necesario un criterio doctrinal. El enseñar, ¿es función del Estado o social? La libertad de enseñanza, ¿es justa o es conveniente? La de cátedra, ¿es lícita?... Importa pues insistir en que nuestro fin es profesional; pero para cumplirlo con perfección, hemos de tener normas para la elección de medios, de caminos para llegar a él, y por eso somos confesionales".

Pasa después a hacer una breve historia de la Confederación, que en sólo tres años ha adquirido un gran desarrollo, y prosigue:

«El mitin del 13 de mayo de 1920 en el teatro de la Zarzuela, de Madrid, el homenaje al Rey en el Teatro Real, el Congreso de Friburgo, la Fiesta del estudiante de 1922, constituyen triunfos ruidosos. De menos brillo, pero quizás de eficacia más durable, fueron el reconocimiento oficial por todas las Universidades de las que se solicitó, durante el régimen autonómico, y el conseguir once de los quince puestos destinados a los estudiantes para la elección del Rector de la Universidad Central» ${ }^{18}$.

De entre las sesiones merecen destacarse por la gran participación y el interés que suscitaron, las referidas a la confesionalidad y a los medios de propaganda, tanto oral como escrita, resaltándose la conveniencia de que

17 Este dato no concuerda con la Memoria... de MARtin ARTAJo, ya que en ésta se dice que el número de asociaciones es de 61; quizá asistieron algunos estudiantes de alguna facultad o escuela universitaria en la que no habia Asociación formalmente constituida, y, por tanto, cuando Artajo elabora el informe esas Asociaciones no le constan.

18 Obras Fecundas..., pp. 35-37. 
cada Federación tuviera su propia revista o periódico escolar. También se trató el tema de la promoción de los Estudiantes Católicos en el Bachillerato. En la última sesión se leyeron las conclusiones ya redactadas definitivamente, que fueron aprobadas por aclamación.

Las conclusiones concretan y desarrollan los temas de estudio propuestos a la Asamblea. Así en la primera conclusión se afirma que:

“La Confederación Nacional de Estudiantes Católicos es una organización escolar profesional. Mantiene su confesionalidad católica, que estima indispensable para el perfecto cumplimiento de sus fines profesionales".

Sobre qué debe ser una Asociación de Estudiantes Católicos, las conclusiones se estructuran en tres apartados: moral, profesional y económico. En la primera conclusión de la parte moral se afirma que se debe promover

"una mayor formación religiosa, social y cultural de los asociados, y en particular de los directores",

lo que se concreta en la tercera conclusión de la siguiente manera:

«También es muy importante la formación de círculos de estudios dirigidos por personas competentes, en las distintas Federaciones, y ayudados en sus trabajos por el Secretariado Confederal de círculos de estudios, que se crea".

Del apartado profesional se destaca una vez más que resulta

"esencial, para la vida de nuestras organizaciones, que dediquen a los asuntos profesionales el mayor interés y esfuerzo, no sólo en su desarrollo después de planteados, sino estudiando iniciativas para llevarlos a la práctica».

Con respecto a la propaganda se decide crear

"el Cuerpo de Propagandistas de la Confederación, nutrido por personal muy selecto. Tendrá régimen especial en todos su aspectos: religioso, profesional, cultural y social. Para ser admitido como propagandista se precisará la asistencia durante un curso, al menos, a un Círculo de Estudios y el ser nombrado por el pleno de la Junta Suprema».

También se recogen unas conclusiones de carácter general en las que se reiteran las posiciones ya defendidas en el mitin de la Zarzuela: defensa de la autonomía universitaria, limitación de la libertad de cátedra para respetar las ideas y creencias religiosas de los alumnos, construcción de 
nuevas Escuelas de enseñanza primaria, reforma del plan de estudios de las Normales realzando la importancia de las prácticas... ${ }^{19}$.

Tras la aprobación de las conclusiones se eligieron los cargos para la Junta Suprema y el Comité Asesor. Para la primera resultó elegido por unanimidad presidente Fernando Martín-Sánchez Juliá; como vicepresidente fue nombrado Juan Bosch Marín, de Valencia, y secretario general José Carbonell. Para el Comité asesor son elegidos Fernando Moreno Ortega, ex-secretario de la Confederación y miembro de la Junta Suprema desde los comienzos, Federico Salmón Amorín, fundador y primer presidente de la Federación Regional Valenciana y miembro de la Junta Suprema durante dos años y José María de la Torre de Rodas, ex-vicetesorero de la Confederación, organizador del Bachillerato y miembro de la Junta Suprema durante un año.

La Asamblea concluyó con unas palabras del recién confirmado presidente, Fernando Martín-Sánchez, y del Rector de la Universidad, Royo Villanova, quien declaró clausurada la Primera Asamblea de la Confederación Nacional de Estudiantes Católicos.

\section{LA CONFEDERACIÓN Y LAS ASOCIACIONES INTERNACIONALES DE ESTUDIANTES}

Como ya hemos comentado anteriormente, el decreto de 21 de mayo de 1919, conocido como decreto Silió por el apellido del ministro de Instrucción Pública que lo sacó adelante, otorgaba a la participación estudiantil un lugar relevante. Esto no hizo sino canalizar el fuerte impulso ideológico que venía de atrás y que se manifestó en el combativo espíritu de los estudiantes españoles.

Los primeros en organizarse y los que contarán con una mayor fuerza serán los Estudiantes Católicos. Otras asociaciones de estudiantes que se formarán en esta época son la Unión Nacional de Estudiantes, laica y de corte liberal, que manifestaba su neutralidad en el tema religioso, por lo que serán llamados "neutros" por los Estudiantes Católicos, y otra asociación de estudiantes socialistas.

Aunque en este trabajo nos vamos a centrar en las asociaciones internacionales católicas, no estará de más reflejar someramente la polémica entre "neutros" y "católicos" con motivo del Congreso de la Federación In-

19 Las conclusiones están recogidas en Obras Fecundas..., pp. 55-63. 
ternacional de Estudiantes, que se desarrolló en Praga a partir del 1 de abril de 1921. Efectivamente, recién terminado el Congreso, algunos oradores, que participaban en un mitin de la Confederación Nacional de Estudiantes Católicos, atacaron duramente a la Unión Nacional de Estudiantes, acusándola de haber impedido su participación en dicho Congreso. Argumentaban que dada la desproporción numérica de afiliados a su favor, les correspondía estar presentes y llevar la representación de los estudiantes españoles en esa reunión ${ }^{20}$. La respuesta fue dada en el Ateneo por una de las participantes en el Congreso de Praga, quien justificaba la no participación de los Estudiantes Católicos por el simple hecho de que los estatutos no permiten el ingreso de asociaciones políticas o confesionales, por lo que

«toda adhesión que los estudiantes españoles quieran enviar al Congreso ha de hacerse por el conducto de la Unión Nacional, que es la única entidad española capacitada legalmente para entenderse con la Confederación Internacionalı2 21.

Pasando ya a estudiar las organizaciones católicas, nos encontramos con que poco después de acabar la Gran Guerra, surgió entre los estudiantes de la Universidad Católica de Friburgo (Suiza) la idea de reunir a todos los universitarios católicos del mundo en una organización, para promover el catolicismo en los medios estudiantiles, $y$, desde ahí, a todos los ámbitos de la vida universitaria, intelectual y social, como medio de fomentar la paz. España colaboró, junto a Suiza y Holanda, en la fundación de la organización.

Así, el 17 de julio de 1921, se encontraron en Friburgo representantes de 20 naciones ${ }^{22}$, que fueron: Alemania, Argentina, Australia, Bélgica, Checoslovaquia, Dinamarca, España, Estados Unidos, Francia, Holanda, Hungría, Inglaterra, Italia, Java, Luxemburgo, Polonia, Portugal, Rumanía, Suiza y Yugoslavia, que representaban a unos cien mil estudiantes, siendo las que contaban con más afiliados España, Estados Unidos, Alemania, Italia y Suiza ${ }^{23}$.

20 Durante el curso 1921-1922 el número de afiliados a los Estudiantes Católicos era de 14651, a la Unión Nacional 1100 y 584 eran independientes. El dato está tomado del Diario de Sesiones del Congreso de los Diputados, de 22 de marzo de 1922, cit. por Martín Artajo en Memoria..., p. 200.

$21 \quad A B C, 19$ de abril de 1921.

22 En el Diario Confederal, incluido en Obras Fecundas..., se habla de 24 naciones participantes; menciona 20 la publicación España en la fundación y en la historia de Pax Romana, 1946. realizada con motivo del XIX Congreso de Pax Romana, segundo que se celebraba en España. También da la cifra de 20 paises el diario La Liberté, de 20 de julio de 1921.

23 Cfr. España en la fundación... 
La sesión inaugural tuvo lugar el 19 de julio de 1921 actuando como presidente el suizo Max Gressly, presidente General de la Sociedad de Estudiantes Suizos y como vicepresidente Fernando Martín-Sánchez. Intervinieron también en el acto inaugural el obispo de Laussana-Ginebra, monseñor Bessón, nombrado más tarde por Benedicto XV Consiliario del Secretariado Internacional de Estudiantes Católicos y M. Perrier, consejero de Estado, que ostentaba la representación del gobierno de la República.

Los días 20 y 21 fueron dedicados a exponer la situación de las distintas asociaciones de cada uno de los países. Se aprobó la creación de un Secretariado Permanente con sede en Friburgo, un Congreso anual de todas las naciones representadas y una Comisión Permanente formada por Martín-Sánchez como presidente, el holandés Bron, el suizo Gressly y una señorita que representaba a las estudiantes asociadas. Se constituyó también una comisión formada por representantes de once naciones encargada de redactar los estatutos; fue nombrado presidente de esta comisión Federico Reparaz, delegado para el extranjero de la Confederación Nacional de Estudiantes Católicos. También se adoptó como lema y nombre de la naciente organización el de Pax Romana.

Fernando Martín-Sánchez fue el encargado de pronunciar el discurso de clausura en el que expuso su deseo de integrar en la organización a los estudiantes hispanoamericanos y subrayó el esperanzador futuro que se abría para Pax Romana, contribuyendo a la pacificación universal a través del diálogo y la convivencia, ya que estaban allí reunidos $-y$ por primera vez juntos tras la guerra alemanes y aliados- los futuros dirigentes de todos esos paises.

No obstante, a pesar del optimismo inicial reflejado en las jornadas posteriores, del que puede ser una buena muestra la siguiente afirmación: La Internacional de los Estudiantes Católicos es un hecho, un hecho completo, una soberbia realidad ${ }^{24}$, en la práctica la Internacional no tuvo una excesiva operatividad, a pesar de su reconocimiento oficial por parte de la Sociedad de Naciones el 4 de febrero de $1923^{25}$.

\section{CONCLUSIONES}

Como hemos tenido oportunidad de comprobar en este estudio, los Estudiantes Católicos fueron ya una realidad al comienzo de los años veinte,

24 "Asi concluye el Congreso de Friburgo", artículo de Federico Reparaz, publicado en El Debate, el 7 de agosto de 1921 .

25 Crr. Obras Fecundas..., p. 92. 
y, por tanto, antes de la llegada de Primo de Rivera. La organización ya estaba creada, tenía una sólida estructura organizativa, una gran implantación en la mayoría de las universidades españolas, un apoyo de la lglesia y de la ACN. de P. y una presencia en las asociaciones católicas internacionales, que permiten hablar de una seria participación en la vida pública española en estas fechas.

Los avatares posteriores, con el del pronunciamiento de Primo de Rivera y la implantación de la Dictadura, permitieron acentuar la influencia de los Estudiantes Católicos en la Universidad, si bien eso resultó contraproducente para la Confederación, ya que la oposición de una buena parte de los estudiantes al dictador, ya en los últimos años del gobierno de éste, harán que pase por un momento difícil, recuperándose en los años de la República, para defender con gran fuerza los principios que la hicieron nacer en 1920. Pero eso es ya otra parte de esta historia. 